\title{
Effective kinetic description of the early-time dynamics in heavy-ion collisions
}

\author{
Naoto Tanji*i \\ Institut für Theoretische Physik, Universität Heidelberg, Philosophenweg 12, 69120 Heidelberg, \\ Germany \\ E-mail: tanjiathphys.uni-heidelberg.de
}

In the idealized high-energy limit of heavy-ion collisions, the system right after a collision is described as an over-occupied gluonic plasma expanding in the longitudinal direction. The understanding of the quark dynamics in such a pre-equilibrium state is of prime importance since it has a direct connection to electromagnetic probes. We report on a numerical study of the Boltzmann equation including two-to-two scatterings of gluons and quarks in the expanding geometry. In the gluon sector, we confirm the self-similar scaling behavior of the one-particle momentum distribution that is consistent with the bottom-up thermalization scenario. Also in the quark sector, we find that the distribution function show scaling behavior like those of gluons.

EPS-HEP 2017, European Physical Society conference on High Energy Physics 5-12 July 2017

Venice, Italy

\footnotetext{
* Speaker.

${ }^{\dagger}$ In collaboration with Raju Venugopalan.
} 
A systematic investigation of the early-time nonequilibrium dynamics of quark-gluon matter in ultrarelativistic heavy-ion collisions is feasible in the limit of weak coupling $\alpha_{s}=g^{2} /(4 \pi) \ll$ 1. Since the typical occupancies of gluons are inversely proportional to the coupling as $f_{\mathrm{g}} \sim$ $1 / g^{2}$ at the early stage of collisions, the system is strongly interacting even though the coupling is weak. The space-time evolution of such overoccupied and strongly-correlated gluon plasma, called Glasma, can be studied by means of real-time classical-statistical simulations of the YangMills equations. Recent 3+1-dimensional Yang-Mills simulations [1] of the expanding Glasma demonstrate that it flows to a nonthermal turbulent fixed point characterized by a self-similar gluon distribution, which is consistent with the bottom-up thermalization scenario of Baier et al. [2].

Compared to the gauge sector, the early-time dynamics of quarks is not well understood although it is a key to understand important issues like the mechanism for chemical equilibration between gluons and light quarks, photons and dilepton production from the early-time nonequilibrium plasma, and possible observable consequences of the chiral magnetic effect. We report on a numerical study of the kinetic equations for the nonequilibrium quark-gluon matter in the longitudinally expanding geometry [3].

The Boltzmann equations for the gluon distribution $f_{\mathrm{g}}$ and the quark distribution $f_{\mathrm{q}}$ in a uniform and longitudinally expanding system take the form of

$$
\left(\frac{\partial}{\partial \tau}-\frac{p_{z}}{\tau} \frac{\partial}{\partial p_{z}}\right) f_{\mathrm{g} / \mathrm{q}}(\tau, \mathbf{p})=C_{\mathrm{g} / \mathrm{q}}\left[f_{\mathrm{g}}, f_{\mathrm{q}}\right]
$$

To the leading order in the coupling, the collision terms $C_{\mathrm{g} / \mathrm{q}}$ contain the effective $1 \leftrightarrow 2$ splitting/merging processes in addition to the $2 \leftrightarrow 2$ scattering processes [4]. In the early-stage such that $Q_{s} \tau \lesssim \alpha_{s}^{-3 / 2}$, however, the $2 \leftrightarrow 2$ scattering processes are expected to play dominant roles according to the bottom-up thermalization scenario [2]. In this study, we consider only the $2 \leftrightarrow 2$ scattering processes. Because of the long-range nature of the interaction in gauge theories, one can apply the small-angle approximation to the collision terms in QCD [5]. By this approximation, the collision terms can be expressed by the sum of a (momentum space) diffusion term and a source term [6],

$$
C_{\mathrm{g} / \mathrm{q}}=-\nabla_{\mathbf{p}} \cdot \mathbf{J}_{\mathrm{g} / \mathrm{q}}+S_{\mathrm{g} / \mathrm{q}} \cdot
$$

The explicit forms of these terms can be found in Refs. [6, 3].

We have numerically solved the kinetic equations (1). The left panel of Fig. 1 shows the time evolution of the transverse momentum distribution for gluons. The initial condition for the gluon distribution is

$$
f_{\mathrm{g}}\left(\tau_{0}, p_{\perp}, p_{z}\right)=\frac{n_{0}}{g^{2}} e^{-\left[p_{\perp}^{2}+\left(\xi_{0} p_{z}\right)^{2}\right] / Q_{s}^{2}}
$$

where $p_{\perp}=\sqrt{p_{x}^{2}+p_{y}^{2}}$ is the transverse momentum. We have used the parameters $g=10^{-3}, n_{0}=$ 0.1 , and $\xi_{0}=2$. Starting from the initial distribution, which is denoted by a black dashed line, the $1 / p_{\perp}$ form is quickly developed at low momenta. In the kinetic framework that includes only the $2 \leftrightarrow 2$ processes, the emergence of the $1 / p_{\perp}$ behavior is a consequence of the transient BoseEinstein condensate. The right panel shows the transverse momentum distribution multiplied by the temporal factor $\left(Q_{s} \tau\right)^{2 / 3}$. By this multiplication, the curves at different times are nicely overlapped 

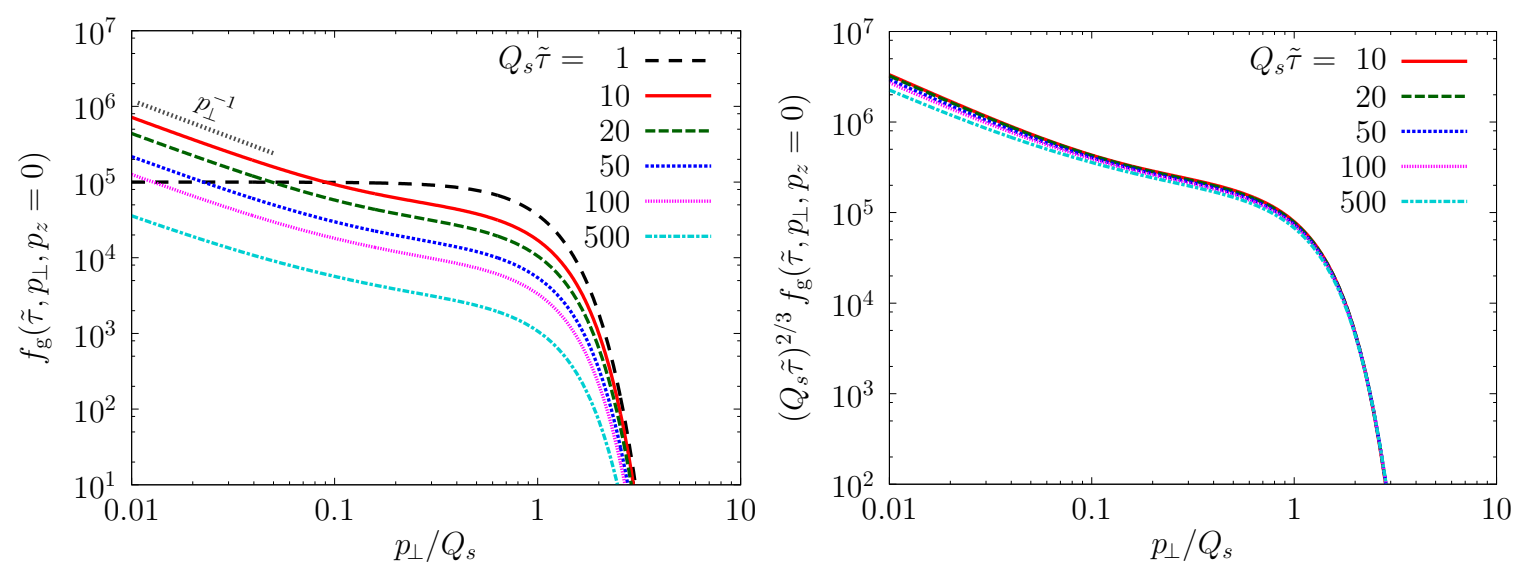

Figure 1: The time evolution of the transverse momentum distribution for gluons evaluated at $p_{z}=0$. Left: original distribution. Right: rescaled distribution.

in the high momentum region $p_{\perp} \gtrsim Q_{s}$. This result as well as another temporal behavior of the longitudinal momentum distribution can be expressed by the following scaling relation:

$$
f\left(\tau, p_{\perp}, p_{z}\right)=\left(Q_{s} \tau\right)^{-2 / 3} f_{S}\left(p_{\perp},\left(Q_{s} \tau\right)^{1 / 3} p_{z}\right),
$$

where $f_{S}$ is a scaling function. This self-similar scaling law is consistent with the bottom-up thermalization scenario [2], and has been demonstrated by large scale classical-statistical real-time lattice simulations [1].

The time evolution of the quark distribution is plotted in the left panel of Fig. 2 as a function of the longitudinal momentum at a fixed transverse momentum $p_{\perp}=Q_{s}$. In the right panel, the distribution multiplied by the factor $\left(Q_{s} \tau\right)^{2 / 3}$ is plotted as a function of $\left(Q_{s} \tau\right)^{1 / 3} p_{z}$. By this rescaling, the data points for different times lie on top of each other except at very small momenta. This result shows that also the quark distribution satisfies the scaling relation (4). The scaling behavior of the quark distribution can be understood as a consequence of the fact that quarks undergo the same small-angle scattering processes as gluons and those scattering processes are Bose-enhanced by final-state gluons. These scaling results have been employed to estimate the photon yields in the
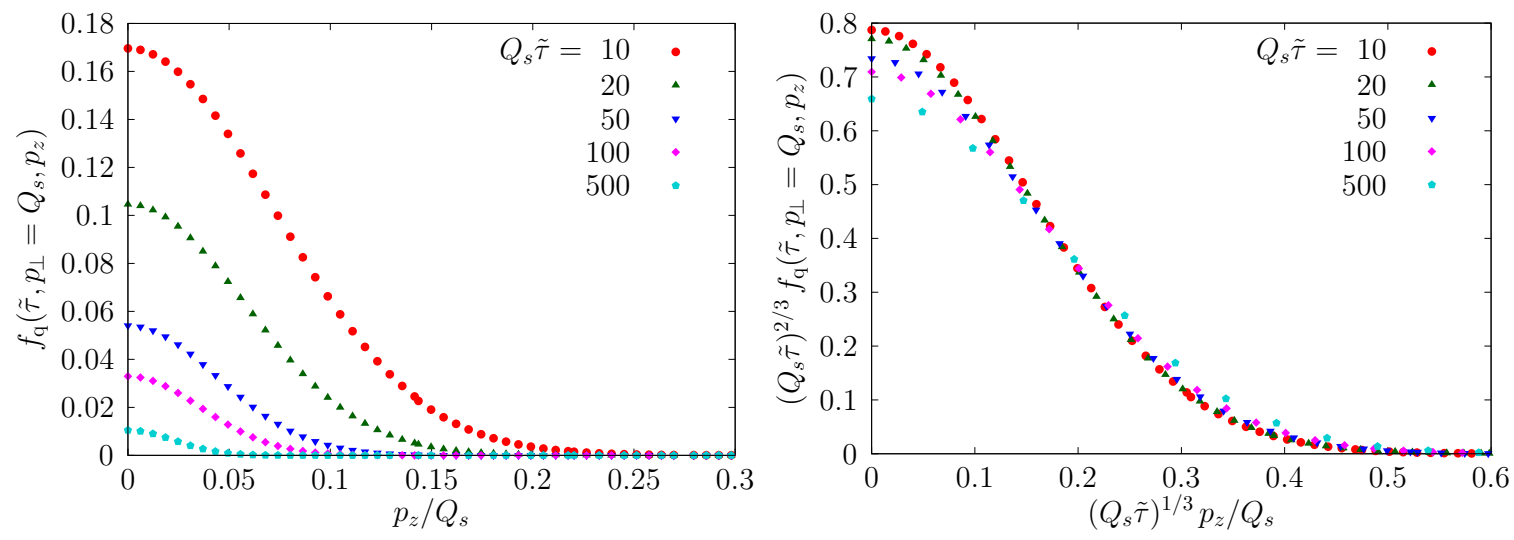

Figure 2: The time evolution of the longitudinal momentum distribution for quarks evaluated at $p_{\perp}=Q_{s}$. Left: original distribution. Right: rescaled distribution. 

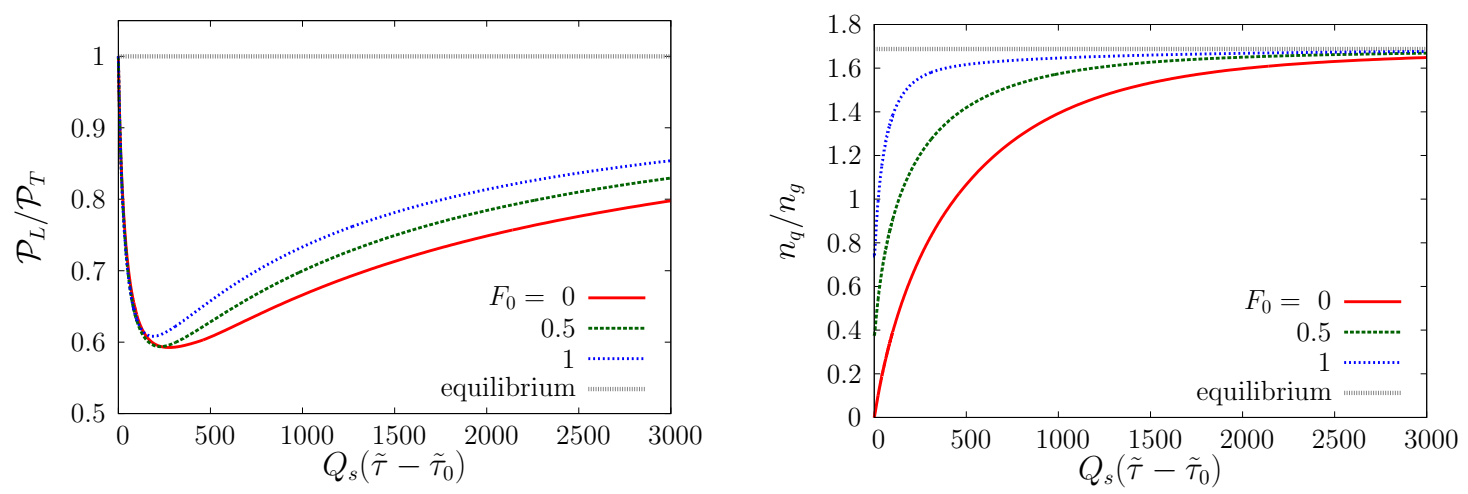

Figure 3: The time evolution of the longitudinal/transverse pressure ratio (left) and the quark/gluon number ratio (right). The values expected in the thermal equilibrium are indicated by black dashed lines.

Glasma, and it has been shown that photon yields from the Glasma are significant relative to those from the thermalized QGP [7].

Lastly, we discuss how the system approaches kinetic and chemical equilibrium in our kinetic framework. Figure 3 shows the time evolution of the longitudinal/transverse pressure ratio (left) and the quark/gluon number ratio (right). The former characterizes kinetic equilibrium and the latter chemical equilibrium. Three different values for the quark initial occupancy $F_{0}$ are compared. A relatively larger value of the coupling $g=0.5$ is taken in these computations. Starting from an isotropic initial condition, the expansion drives the system anisotropic at early times. Later, the pressure ratio turns to increase because of interaction. However, the approach to the equilibrium value is rather slow as the only $2 \leftrightarrow 2$ scatterings are considered in these calculations. In contrast, the number ratio approaches its equilibrium value more quickly. This is because the equipartition is not hindered by the expansion as both quarks and gluons experience the expansion.

\section{References}

[1] J. Berges, K. Boguslavski, S. Schlichting and R. Venugopalan, Turbulent thermalization process in heavy-ion collisions at ultrarelativistic energies, Phys. Rev. D89 (2014) 074011, [1303.5650].

[2] R. Baier, A. H. Mueller, D. Schiff and D. T. Son, 'Bottom up' thermalization in heavy ion collisions, Phys. Lett. B502 (2001) 51-58, [hep-ph/ 0009237 ].

[3] N. Tanji and R. Venugopalan, Effective kinetic description of the expanding overoccupied Glasma, Phys. Rev. D95 (2017) 094009, [1703.01372].

[4] P. B. Arnold, G. D. Moore and L. G. Yaffe, Effective kinetic theory for high temperature gauge theories, JHEP 01 (2003) 030, [hep-ph/ 0209353 ].

[5] A. H. Mueller, The Boltzmann equation for gluons at early times after a heavy ion collision, Phys. Lett. B475 (2000) 220-224, [hep-ph/9909388].

[6] J.-P. Blaizot, B. Wu and L. Yan, Quark production, Bose-Einstein condensates and thermalization of the quark-gluon plasma, Nucl. Phys. A930 (2014) 139-162, [1 402.5049 ].

[7] J. Berges, K. Reygers, N. Tanji and R. Venugopalan, Parametric estimate of the relative photon yields from the glasma and the quark-gluon plasma in heavy-ion collisions, Phys. Rev. C95 (2017) 054904, [1701.05064]. 